\title{
TOPOLOGICAL METHODS IN THE THEORY OF LEBESGUE AREA
}

\section{J. W. T. YOUNGS}

1. Introduction. In conformity with the title of this address, the principal objective is the consideration of topological techniques which have been employed in the theory of surface area initiated by Lebesgue $[15]^{1}$ in his thesis.

It may appear strange that topology is required at all in a discipline classified in the field of analysis-certainly Lebesgue in 1902 gave little hint of what was to come. On the other hand, it cannot be claimed that this sort of thing is an isolated phenomenon, and perhaps one may be permitted to argue that one of the beauties of mathematics is the presence of such mixtures between fields.

Historically speaking, it was de Geöcze who called attention to certain phenomena-in a series of highly significant papers written during the years 1908 to 1914 - and, in attempting to fit these phenomena into the framework of the theory, first noticed the need for topology. (For a partial list of these papers see de Geöcze [12]. A more detailed bibliography at this point, and elsewhere throughout the paper, is found in Radó [26].)

In discussing this matter let it be said, even at the risk of considerable over-simplification, that the significant problems in Lebesgue area have their source in the attempt to find 2-dimensional analogues for four classical results in the theory of length. In any event, with an eye on cohesion, it is here planned to consider only those topological techniques which have bearing on what might be called the analogy problem.

In consequence of this general plan of attack it is not only appropriate to sketch these classical results, but fortunately in so doing one automatically gains a measure of intuition by analogy and adequate motivation is provided for the 2-dimensional theory.

Before plunging into the discussion, however, a remark should be made as to the manner of presentation in contradistinction to the plan of attack. Matters of analogy leading to intuitive understanding are considered important enough to develop so as to be practically self-contained. (For example, during the first part of this paper every

An address delivered before the Summer Meeting of the Society on September 9, 1948, by invitation of the Committee to Select Hour Speakers for Annual and Summer Meetings; received by the editors December 8, 1948.

1 Numbers in brackets refer to the bibliography at the end of the paper. 
effort will be made to define the principal concepts involved.) Consequently, it is to be hoped that a substantial portion of the subject matter will be followed readily by all. This plan must, of course, be abandoned in those sections dealing with specific topological techniques-this is certainly not the place to consider such matters in any detail.

2. Coordinate functions. For the purpose of considering the previously mentioned four classical results for curves, suppose $f$ is a mapping, that is, a continuous transformation, from the unit interval $X^{1}$ into Euclidean 3-space $E^{3}$. It is assumed that coordinate axes $Y^{1}, Y^{2}$, and $Y^{3}$ have been selected in $E^{3}$. If $p^{i}: E^{3} \rightarrow Y^{i}$ is the notation for the perpendicular projection of $E^{3}$ onto $Y^{i}$, while $f^{i}$ is the composition $p^{i} f$, then $f^{i}: X^{1} \rightarrow Y^{i}, i=1,2,3$, and in analysis the mapping $f$ is usually given by the so-called coordinate functions:

$$
y^{1}=f^{1}(x), \quad y^{2}=f^{2}(x), \quad y^{3}=f^{3}(x), \quad x \in X .
$$

3. Classical formula. The classical integral formula $\int_{X^{1}}\left[\left(J^{1}\right)^{2}\right.$ $\left.+\left(J^{2}\right)^{2}+\left(J^{3}\right)^{2}\right]^{1 / 2} d x$, where $J^{i}=d f^{i} / d x, i=1,2,3$, is to be designated by $I^{1}(f)$. This formula will not generally provide the length of the "curve given by $f$." Indeed, even if Lebesgue integration is used-and this will invariably be the case-the formula will generally be a meaningless collection of marks, since there is no guarantee that the integrand exists almost everywhere and is summable. In this connection it is to be understood, however, that if the symbol $I^{1}(f)$ appears in a statement then it has meaning; in other words, the integrand in question does exist almost everywhere and, indeed, is summable.

4. Representation. Before going any farther, it is advisable to make some comment on the phrase "curve given by $f$ " used in $\$ 3$.

The mapping $f$ is simply a representation for a curve and the concept of changing the representation is common, even in elementary mathematics, though it is subject to many interpretations. For a number of reasons the most convenient interpretation for present purposes is due to Fréchet [11]. Accordingly $f: X^{1} \rightarrow E^{3}$ and $g: X^{1} \rightarrow E^{3}$ are said to represent the same curve, or, to use the modern terminology, are said to be Fréchet equivalent (notation: $f \sim g$ ), if and only if for every $\epsilon>0$ there is a homeomorphism $h_{\varepsilon}: X^{1} \approx X^{1}$ such that $d\left\{f(x), g h_{\epsilon}(x)\right\}<\epsilon, x \in X^{1}$. A formal definition for a curve can now be offered in terms of this equivalence relation. (See Youngs [31] where the matter is considered in detail for both curves and surfaces.)

It is well known that the length of a curve is initially defined in terms of a given representation as the limit of the lengths of any 
sequence of inscribed polygonal mappings which converge uniformly to the given representation. It is easy to see, however, that the length of a curve is-as it should be-independent of the representation. (For greater detail see Radó [26].) The notation $L^{1}(f)$ is here employed for the length of the curve given by the mapping $f: X^{1} \rightarrow E^{8}$.

5. Classical theorems for curves and future criteria for surfaces. It is now possible to state three of the promised classical results:

$\mathrm{C}_{1}: L^{1}(f)<\infty \leftrightarrow f^{i}$ is $\mathrm{BV}$ (=of bounded variation), $i=1,2,3$.

$\mathrm{C}_{2}: L^{1}(f)<\infty \rightarrow L^{1}(f) \geqq I^{1}(f)$, and $L^{1}(f)=I^{1}(f) \leftrightarrow f^{i}$ is AC (=absolutely continuous), $i=1,2,3$. (In conformity with the convention in $\$ 3$ the first statement means: $L^{1}(f)<\infty \rightarrow I^{1}(f)$ exists and $L^{1}(f) \geqq I^{1}(f)$.)

$C_{3}: L^{1}(f)<\infty \rightarrow$ there is a representation $g \sim f$ such that $I^{1}(g)=L^{1}(g)$ $=L^{1}(f)$.

6. Interval function. It would be presumptuous to define the concepts $\mathrm{BV}$ and $\mathrm{AC}$ except for the fact that a record of these definitions affords both an insight into the 2-dimensional case and a considerable economy in the sequel.

For convenience suppose $\phi: X^{1} \rightarrow E^{1}$ is a mapping from the unit interval into a line. (The mapping $\phi$ is of the same type, therefore, as the coordinate functions $f^{1}, f^{2}$, and $f^{3}$ of $\$ 2$.)

The key to the definitions of $\mathrm{BV}$ and $\mathrm{AC}$ is a certain interval function now to be considered. Suppose that $r$ is a closed interval $a \leqq x \leqq b$ in $X^{1}$, while $\dot{r}=a \cup b$. Then $v_{\phi}(r)$, the interval function in question, is simply the length of the interval from $\phi(a)$ to $\phi(b)$ in $E^{1}$.

7. An alternative interpretation. For the sake of insight into the 2-dimensional case another interpretation of $v_{\phi}(r)$ is important.

Consider the components (=maximal connected subsets) of $E^{1}$ $-\phi(\dot{r})$, and to avoid trivialities assume there is a bounded component $K$. Then

$$
v_{\phi}(r)=|K|
$$

where $|K|$ is the 1-dimensional Lebesgue measure of $K$; in this case, simply the length of the open interval $K$.

Let $\Gamma$ be the family of those mappings $\gamma: r \rightarrow E^{1}$ which agree with $\phi$ on $\dot{r}$. Then

$$
\gamma(r) \supset K \cup \phi(\grave{r}), \quad \gamma \in \Gamma,
$$

but there is a mapping $\gamma_{0} \in \Gamma$ (for example the unique linear mapping in $\Gamma$ ) such that

$$
\gamma_{0}(r)=K \cup \phi(\dot{r}) .
$$


Hence

$$
\bigcap_{\Gamma} \gamma(r)=\gamma_{0}(r)
$$

Therefore, $\left[E^{1}-\phi(\grave{r})\right] \cap \bigcap_{\Gamma} \gamma(r)=\left[E^{1}-\phi(\grave{r})\right] \cap \gamma_{0}(r)=\gamma_{0}(r)-\phi(\grave{r})=K$, and one has the interpretation

$$
v_{\phi}(r)=\left|\left[E^{1}-\phi(\dot{r})\right] \cap \bigcap_{\Gamma} \gamma(r)\right| .
$$

8. Bounded variation and absolute continuity. If $R$ is a closed interval in $X$, let $\sigma(R)$ be the notation for a subdivision of $R$; that is, a finite collection of $r$ 's, whose union is $R$ and whose interiors are disjoint in pairs. Define

$$
V_{\phi}(R)=\sup _{\sigma(R)} \sum_{r \in \sigma(R)} v_{\phi}(r) .
$$

The number $V_{\phi}(R)$ is called the variation of $\phi$ on $R$, and

$$
\phi \text { is } \mathrm{BV} \text { means } V_{\phi}\left(X^{1}\right)<\infty \text {. }
$$

As for the concept of $\mathrm{AC}$, to do minor violence to the customary definition:

$\phi$ is AC means: (a) for every $\epsilon>0$ there is a $\delta(\epsilon)$ such that if $r_{1}, \cdots, r_{n}$ is a set of closed intervals with interiors disjoint in pairs and $\sum\left|r_{i}\right|<\delta(\epsilon)$, then $\sum V_{\phi}\left(r_{i}\right)<\epsilon$.

$$
V_{\phi}(R)=\sum_{r \in \sigma(R)} V_{\phi}(r), \text { for any } \sigma(R) .
$$

It is well known, however, that (b) is always true, hence it is redundant in this definition and is here displayed only for future use.

9. Final classical theorem for curves and future criterion for surfaces. Now that these definitions have been recorded it is important to observe that in the case of a mapping $f: X^{1} \rightarrow E^{3}$, if $\sigma$ is a subdivision of $X^{1}$ and

$$
g_{\sigma}(f)=\sum_{r \in \sigma}\left[v_{f^{1}}^{2}(r)+v_{f^{2}}^{2}(r)+v_{f^{3}}^{2}(r)\right]^{1 / 2},
$$

then $g_{\sigma}(f)$ is the length of the inscribed polygon determined by $\sigma$. Moreover, if

$$
G(f)=\sup _{\sigma} g_{\sigma}(f)
$$

then one has the final classical result:

$\mathrm{C}_{4}: L^{1}(f)=G(f)$.

(Notice that $\mathrm{C}_{4}$ implies $L^{1}(\phi)=G(\phi)=V_{\phi}\left(X^{1}\right)$.) 
10. Surfaces. In now considering the situation for surfaces, the same general outline is employed except that $f$ is now a mapping from the unit square $X^{2}$ into Euclidean 3-space $E^{3}$, and in contradistinction to the earlier convention, it is now assumed that $Y^{1}, Y^{2}$, and $Y^{3}$ are coordinate planes in $E^{3}$. If $p^{i}: E^{3} \rightarrow Y^{i}$ is the perpendicular projection, then let $f^{i}=p^{i} f$ as before. The mapping $f^{i}: X^{2} \rightarrow Y^{i}$ is a so-called flat transformation from the unit square into a plane, $i=1,2,3$.

The classical integral formula (see \$3) remains unchanged except that $J^{i}$ is now the Jacobean of the flat transformation $f^{i}$ and, of course, 2-dimensional integration is understood. The formula will be designated by $I^{2}(f)$ and the earlier conventions concerning its use apply. (See \$3.)

It has been stated that outstanding problems in Lebesgue area arise in the search for definitions of $\mathrm{BV}$ and $\mathrm{AC}$ for flat transformations which will provide results analogous to the classical theorems $C_{1}, C_{2}$, $\mathrm{C}_{3}$, and $\mathrm{C}_{4}$ (see $\$ \$ 5$ and 9). These theorems thus become criteria for the suitability of proposed concepts of $B V$ and $A C$.

Initial attacks on the problem were made by de Geöcze, and though he was far from being entirely successful in his venture, it came to be considered a safe judgment-even in a field where history has shown the most plausible conjectures to be fraught with disaster-that modifications of his ideas would stand the test of these criteria. In a sense, appropriate concepts have long been available-the difficulty has been in providing proofs.

In defining $\mathrm{BV}$ and $\mathrm{AC}$ for flat transformations, the development will be by analogy from the 1-dimensional case and it must be stated that considerable expository license will be taken with the ideas of Cesari $[3,4,5,6,7]$, Radó $[18,19,20,24,25,26,27]$, and Reichelderfer $[27,28]$.

In accord with the modifications introduced above, the mapping $\phi$ (cf. \$6) is now considered to be a flat transformation from the unit square $X^{2}$ into a plane $E^{2}$. (It is therefore of the same type as the mappings $f^{i}$ found above.) The interval function of the 1-dimensional case is to be replaced by a function of the closure $r$, of a Jordan region bounded by a simple closed polygon $r \subset X^{2}$.

In the case for curves $v_{\phi}(r)$ was interpreted as the 1-dimensional measure of a certain set in $E^{1}-\phi(\dot{r})$ (see $\left.\$ 7\right)$. By direct analogy, $v_{\phi}(r)$ is now defined to be the 2-dimensional measure of the corresponding set $\left[E^{2}-\phi(\dot{r})\right] \cap \bigcap_{\Gamma} \gamma(r)$ where $\Gamma$ is the family of mappings $\gamma: r \rightarrow E^{2}$ which agree with $\phi$ on $\dot{r}$.

The variation $V_{\phi}(R)$ of $\phi$ on $R$ is defined in exactly the same manner (see \$8) and there is no change in the definitions of $B V, A C$, and $G(f)$. 
(See $\S \S 8$ and 9.) In view of this fact these definitions are not recorded anew. It is to be noted, however, that item (b) in the definition of AC is not redundant. In other words, what was really a theorem in the 1-dimensional case now becomes an essential portion of the definition of AC. (See Cesari [6].)

It should be noted that the functional $g_{\sigma}(f)$ can no longer be interpreted as the area of an inscribed polyhedron even if $\sigma$ is a triangulation of $X^{2}$. The functional $G(f)$, however, may be interpreted as one of the possible definitions for the de Geöcze area of the surface represented by $f$.

11. Lebesgue area. Prior to considering how these concepts fit into the framework of the criteria $\mathrm{C}_{1}, \mathrm{C}_{2}, \mathrm{C}_{3}$, and $\mathrm{C}_{4}$ (see $\S \S 5$ and 9), it is proper to define $L^{2}(f)$, the Lebesgue area of the surface represented by $f$. A mapping $\mathfrak{p}: X \rightarrow E^{3}$ is said to be polyhedral (=quasi linear, see McShane [16]) if there is a triangulation $T$ of $X$ with the property that $\mathfrak{p}^{i}=p^{i} \mathfrak{p}$ is linear on each triangle of $T$. (See $\$ 10$.) The elementary area $E(p)$ of the polyhedron given by $p$ is simply the sum of the areas of the triangles $\mathfrak{p}(\Delta), \Delta \in T$, and

$$
L^{2}(f)=\inf \left[\liminf _{n \rightarrow \infty} E\left(\mathfrak{p}_{n}\right)\right],
$$

where the infimum is taken for all sequences $\mathfrak{p}_{n}$ converging uniformly to $f$. It is not difficult to see that if $f$ and $g$ represent the same surface (see $\S 4$ ) then $L^{2}(f)=L^{2}(g)$. (There is an obvious lack of analogy here between the definitions of length (see $\$ 4$ ) and area. In point of fact, length could equally well have been defined by 1-dimensional considerations precisely analogous to the 2 -dimensional considerations above. This was not done since the definition of $\$ 4$ is better known. It is not to be supposed, conversely, that area can be defined by considerations precisely analogous to those employed in the definition of length given in $\$ 4$. For a discussion of this matter see Youngs [31].)

12. Generalized Jacobians. In addition to the definition of Lebesgue area, it is necessary to say a word about the classical integral formula $I^{2}(f)$, where the symbols $J^{i}$ now stand for the Jacobian of $f^{i}, i=1,2,3$. (See $\S \S 3$ and 10.) For a variety of reasons it is necessary to generalize the concept of Jacobian.

It will be recalled that the absolute value of the ordinary Jacobian of a flat transformation $\phi: X^{2} \rightarrow E^{2}$ measures the local "ratio of magnification" accorded by the mapping. By analogy from the 1-dimensional case (see end of $\$ 9$ ) one can hope that $V_{\phi}(r)=L^{2}(\phi \mid r)$-where 
$\phi \mid r$ is the mapping $\phi$ considered only on $r$. Hence a natural generalization is to define

$$
\mathcal{F}(x)=\lim _{|s| \rightarrow 0} \frac{V_{\phi}(s)}{|s|}
$$

where $s$ is a square with sides parallel to the axes of $X^{2}$ and $x \in s^{0}$. (If the ordinary Jacobian $J(x)$ exists almost everywhere then the same is true of $\mathcal{F}(x)$, and $|J(x)|=\mathcal{F}(x)$ almost everywhere; see Cesari [4] and Radó [26].)

If the ordinary Jacobians in the classical integral formula $I^{2}(f)$ are replaced by the generalized Jacobians, then the new formula is designated by $\Im^{2}(f)$ and the earlier conventions concerning its use apply (see $\$ \S 3$ and 10 ).

13. Results for surfaces. As to the four criteria (see $\$ \S 5$ and 9) it is now possible to state that these statements all hold if $I^{1}$ is replaced by $\Im^{2}$ and $L^{1}$ by $L^{2}$.

This simple assertion is by no means simple to prove. It represents the concerted efforts of many mathematicians over approximately half a century and, in point of fact, $\mathrm{C}_{\mathbf{3}}$ has only recently been announced by Cesari [8].

In proving these theorems one must naturally work with the basic function $v_{\phi}(r)$, and it must be stated that an equivalent definition in terms of the degree of a mapping is employed. (See, for example, Cesari [3] and Radó [26].) This equivalent definition need not concern us. The point is that in consequence of this fact, it is only to be expected that standard theorems on the degree will be encountered. What is perhaps unexpected is that further topological methods, to be known in this context as topological adventures, must be brought to bear on the situation. In any event it is the topological adventures alone which will be the center of attention.

It should also be stated that in view of technical complexities involved in the proofs and, indeed, the sheer lengths of the proofs themselves, no details can be undertaken, and the application of the topological adventures to be considered can at best be implied.

14. Inequality of Cesari and criterion $C_{1}$. Of the four theorems $C_{1}$ perhaps deserves special attention in view of its difficulty and the bearing its solution has on some of the others, notably $\mathrm{C}_{4}$.

That $L^{2}(f)<\infty$ implies $f^{i}$ is BV was shown independently by Cesari [3] and Radó [24] during the war years. Cesari, however, proved both parts of $\mathrm{C}_{1}$ using an argument which culminated in the basic inequality, 


$$
V_{f^{i}}\left(X^{2}\right) \leqq L^{2}(f) \leqq V_{f^{1}}\left(X^{2}\right)+V_{f^{2}}\left(X^{2}\right)+V_{f^{3}}\left(X^{2}\right), \quad i=1,2,3 .
$$

From this inequality the issue is settled at a glance. This superb result must certainly rank as an accomplishment of the first magnitude.

The right half of the Cesari inequality is much more difficult to prove than the left. On the other hand, it will follow immediately from the definition of $L^{2}(f)$ if it can be shown that for each integer $n$ there is a polyhedral mapping $\mathfrak{p}_{n}: X \rightarrow E^{3}$ such that

$$
\begin{array}{cr}
d\left(f(x), \mathfrak{p}_{n}(x)\right)<1 / n, & x \in X . \\
E\left(\mathfrak{p}_{n}\right) \leqq V_{f^{1}}\left(X^{2}\right)+V_{f^{2}}\left(X^{2}\right)+V_{f^{3}}\left(X^{2}\right) . & (\text { See } \$ 11 .)
\end{array}
$$

To obtain (a) by itself is a triviality, but to obtain it in conjunction with (b) is no easy task and involves carefully fitting together a polygonal mapping by a highly delicate argument. A salient step deeply imbedded in the chain of reasoning is concerned with a property of Euclidean 3-space which should be of some interest.

In $E^{3}$ let $M$ consist of the coordinate axes $X, Y, Z$. With $\delta>0$, define $M_{\delta}$ to be the union of the four lines:

$$
\begin{array}{ll}
X_{\delta}: y=0, z=-\delta . & Y_{\delta}: x=0, z=\delta . \\
Z_{\delta}^{+}: x=\delta, y=\delta . & Z_{\delta}^{-}: x=-\delta, y=-\delta .
\end{array}
$$

Now suppose that one is given a closed path in $E^{3}-M$ at a distance greater than $\delta$ from $M$. A lemma of Cesari [2] states that if the path is contractible in $E^{8}-M_{\delta}$ then it is also contractible in $E^{3}-M$ and conversely. (This form of the statement is due to Eilenberg [10] who has also considerably shortened the original proof.)

The inequality of Cesari shows, by the way, that

$$
L^{2}\left(f^{i}\right)=V_{f^{i}}\left(X^{2}\right), \quad i=1,2,3,
$$

and hence

$$
L^{2}\left(f^{i}\right) \leqq L^{2}(f) \leqq L^{2}\left(f^{1}\right)+L^{2}\left(f^{2}\right)+L^{2}\left(f^{3}\right), \quad i=1,2,3 .
$$

But (a) can be proved independently, and hence the Cesari inequality follows if the same is true of (b).

The first half of (b) is obvious and the second has the deceptive air of appearing to be simpler to prove than the Cesari inequality since the functional $L^{2}$ alone appears. However, it is not known whether (b) can be proved directly or not.

15. Middle space topology. It is not to be supposed however that $\mathrm{C}_{1}$ was proved without further topological adventures. For example a close scrutiny of the character and disposition of the inverse sets 
$f^{-1}(y), y \in E^{3}$, was required. Since studies of this character also appear in connection with the other theorems, and here analytic topology plays a major role, the matter cannot be dismissed with cursory mention. (For reasons which will become apparent this whole branch of the theory has aptly been called middle space topology by Radó [23].)

The basic concept involved is that of a monotone-light factorization of a mapping. If $f: X \rightarrow Y$ is a mapping from a compact space into a metric space, then the Eilenberg-Whyburn Factor Theorem (see Whyburn [30]) states that there is a pair of mappings $m: X \rightarrow \mathbb{X}$ and $l: \mathfrak{X} \rightarrow Y$ such that:

(a) $f=l m$.

(b) $m$ is monotone $\left(=m^{-1}(x)\right.$ is a continuum, $\left.x \in X\right)$, and $m(X)=\mathfrak{X}$.

(c) $l$ is light (=no component of $l^{-1}(y)$ consists of more than one point, $y \in Y$ ).

The space $\mathfrak{X}$ is called the middle space of the monotone-light factorization $l m$ of $f$.

In the study of Lebesgue area it became evident that the structure of the middle space $\mathfrak{X}$ - the image of the monotone factor-was of considerable importance in contradistinction to the apparently paradoxical fact that the structure of $f(X)$-the image of $f$-had relatively little to do with the matter.

For example, it was observed by Morrey [17] that if $X$ is a 2-cell and $L^{2}(f)<\infty$ then it is possible to find a new representation $g: X \rightarrow E^{3}$ such that the Lebesgue area is given by the classical integral formula $I^{2}(g)$.

16. Representation problem. This is perhaps as good a time as any to state that the general problem of finding new representations for a surface had earlier attracted the attention of Kerékjártó [14]. He considered the case in which $X$ is a 2-sphere while $f_{1}: X \rightarrow E^{3}$ and $f_{2}: X \rightarrow E^{3}$ are representations of the same surface, that is to say, are Fréchet equivalent (see \$4). Under these conditions, Kerékjártó showed that there is a monotone-light factorization $l m_{i}$ of $f_{i}$ with common middle space $\mathfrak{X}, i=1,2$. (Note that the factorizations also have a common light factor.)

It was initially supposed that the converse of this theorem is true. This is not the case (see Youngs [32]), and it should be mentioned that it is the converse which is important in these problems. However, Kerékjárto's work initiated the search for conditions which are both necessary and sufficient for $f_{1} \sim f_{2}$. (See $\$ 4$.) This is the representation problem for surfaces. The solution requires the use of algebraic topology - in this presentation the Cech cohomology theory with the additive group of integers as the coefficient group will be 
employed. The result in question is the following and is initially stated for the case $X$ is a 2-cell with boundary $\dot{X}$.

$f_{1} \sim f_{2}$ if and only if:

1. There is a monotone-light factorization $l m_{i}$ of $f_{i}$ with common middle space $\mathfrak{X}, i=1,2$.

2. If $\dot{m}_{i}=m_{i} \mid \dot{X}$ then there is a monotone-light factorization $\lambda \mu_{i}$ of $\dot{m}_{i}$ with common middle space $\Xi, i=1,2$. (This implies $m_{1}(\dot{X})=m_{2}(\dot{X})$ $\equiv \dot{X}$.

3. If $m_{i}^{*}: H^{2}(\dot{X}, \dot{X}) \rightarrow H^{2}(X, \dot{X})$ and $\mu_{i}^{*}: H^{1}(\Xi) \rightarrow H^{1}(\dot{X})$ are the homomorphisms induced by the mappings $m_{i}$ and $\mu_{i}$ respectively, $i=1,2$, then there is an automorphism $\eta: H^{2}(X, \dot{X}) \approx H^{2}(X, \dot{X})$ such that with respect to the following diagram

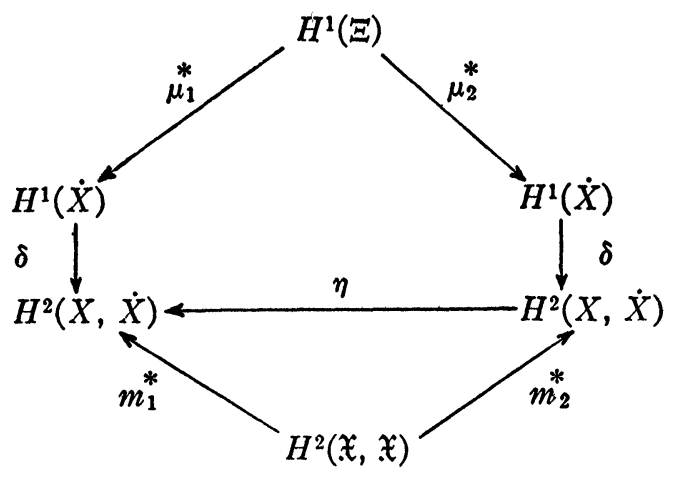

one has $\delta \mu_{1}^{*}=\eta \delta \mu_{2}^{*}$ and $m_{1}^{*}=\eta m_{2}^{*}$.

In the event $X$ is a 2 -sphere, $\dot{X}=0$, and the statement of the result is simplified in the obvious manner (Youngs [32]).

17. Additivity theorem. The first active use made of the solution of the representation problem was in the proof of an additivity theorem for the Lebesgue area. Suppose $f: X \rightarrow E^{3}$ represents a surface with $X$ a 2 -sphere, and $l m$ is a monotone light factorization of $f$ with middle space $\mathfrak{X}$. (See $\$ 15$.) The collection of true cyclic elements of $\mathfrak{X}$ is at most denumerable. (See Whyburn [30].) Let them be represented by $\mathbb{E}_{1}, \mathbb{E}_{2}, \mathbb{E}_{3}, \ldots$. It is well known that there is a unique monotone retraction $r_{n}: \mathfrak{X} \rightarrow \mathbb{E}_{n}, n=1,2,3, \ldots$.

If $f_{n}=l r_{n} m, n=1,2,3, \cdots$, then this class of mappings is said to be the cyclic decomposition of $f$, and the cyclic additivity theorem for Lebesgue area states that

$$
L^{2}(f)=\sum L^{2}\left(f_{n}\right),
$$

where the symbols on the right are interpreted to mean 0 if there are no true cyclic elements. 
It should be stated that the theorem is also true in the event $X$ is a 2-cell as has been shown by Radó [26] and Helsel [13].

The cyclic additivity theorem shows that $L^{2}(f)=0$ if the middle space has no true cyclic elements, that is to say, if $\mathfrak{X}$ is a dendrite. Rado [22] has also proved the converse of this theorem thus providing an elegant characterization for surfaces of zero area.

18. Criterion $\mathbf{C}_{4}$. It can be shown that the Geöcze area is also cyclicly additive, and hence the equality of the two definitions of area, that is, a solution of $\mathrm{C}_{4}$ (see $\S 9$ ) follows if $G\left(f_{n}\right)=L^{2}\left(f_{n}\right)$, $n=1,2,3, \ldots$ This has been done independently by Cesari $[5,7]$ and Radó $[24,26]$ in the event $L\left(f_{n}\right)<\infty$-it is topologically interesting to note that the proof depends heavily on the fact that the surface represented by $f_{n}$ can also be represented by a mapping $g_{n}$ which is light on the interior of $X^{2}, n=1,2,3, \cdots$.

In the event $L^{2}\left(f_{n}\right)=\infty$ Cesari completed the solution of this celebrated problem by employing his inequality (see \$14) together with the obvious fact that

$$
V_{f_{n}}^{i}\left(X^{2}\right) \leqq G\left(f_{n}\right), \quad i=1,2,3 .
$$

For now

$$
L\left(f_{n}\right) \leqq V_{f_{n}}^{1}\left(X^{2}\right)+V_{f_{n}}^{2}\left(X^{2}\right)+V_{f_{n}}^{8}\left(X^{2}\right) \leqq 3 G\left(f_{n}\right) .
$$

Consequently $L^{2}\left(f_{n}\right)=\infty$ implies $G\left(f_{n}\right)=\infty$.

19. Criteria $\mathbf{C}_{2}$ and $\mathbf{C}_{3}$. The lack of recent specific mention of $\mathrm{C}_{2}$ and $\mathrm{C}_{3}$ (see $\$ 5$ ) may lead one to suppose that here, at any rate, no topological adventures are required. While, relative to the other theorems, there is a certain element of truth to this surmise, it is not to be supposed that $\mathrm{C}_{2}$ or $\mathrm{C}_{3}$ can be proved in the body of analysis. For example, a basic step in the proof of $\mathrm{C}_{2}$ is the fact that $\mathrm{C}_{4}$ is true in particular if $L^{2}(f)<\infty$. (See Cesari [4] and Radó [26].) Hence indirectly at least the most powerful methods of middle space topology are required. As for $\mathrm{C}_{3}$, Cesari's announcement shows that he proves this by first generalizing the result of Morrey already stated in part. (See $\$ 15$ and Cesari $[8,9]$.) The argument again may be based upon middle space topology. Hence in a certain sense the most that can be said is that no topological adventures are required which are spectacularly different from those already employed in the proofs of $\mathrm{C}_{1}$ and $\mathrm{C}_{4}$.

Relative to $\mathrm{C}_{3}$, a portion of the results of Cesari shows that if $L^{2}(f)<\infty$ then there is a closed set $H \subset X$ and a representation $g$ of the surface such that $L^{2}(g)=\int_{H}\left[\left(J_{8}^{1}\right)^{2}+\left(J_{8}^{2}\right)^{2}+\left(J_{8}^{8}\right)^{2}\right]^{1 / 2} d x$. (See $\$ 3$ and 
Cesari [8].) The subscript $s$ is here employed to indicate that the ordinary Jacobians are taken relative to the representation $g$ but that the concept of differentiation is appropriately generalized using the work of Stepanoff.

To date this result is the closest approach to an unsolved problem of obvious interest. If $L^{2}(f)<\infty$ is there a representation $g \sim f$ (see $\$ 4$ ) such that $L^{2}(g)=I^{2}(g)$ ? In other words is every surface of finite Lebesgue area so representable that the ordinary classical integral formula applied to this representation yields the Lebesgue area? Radó [21] has shown that if $I^{2}(f)$ has meaning, then $L^{2}(f) \geqq I^{2}(f)$, and it is known that every surface, regardless of its Lebesgue area, has a representation $g$ for which $I^{2}(g)=0$. (See Youngs [33].) If the above question is answered in the affirmative it will certainly provide a beautiful link between the classical ideas of the last century and the modern theory of Lebesgue area. (Added in proof: Cesari has recently shown that such representations exist.)

20. Generalizations. The statement of this unsolved problem concludes this survey of some of the topological adventures forced by these classical problems. However, a few concluding remarks will be made on generalizations in two directions:

1. The problem of mappings from cells of dimension higher than 2 .

2. The problem of mappings from compact connected 2-manifolds.

The first problem has attracted the attention of Federer who has reported (in correspondence) a fruitful generalization of the concept of BV for mappings from an $n$-cell into Euclidean $n$-space. His concept is, in point of fact, a modification of the method by which Radó defines BV in the 2-dimensional case; namely by using the concept of essential multiplicity which he brought to bear on these problems twenty years ago. (See Radó [18].)

Without going into details, Federer's ideas may be sketched as follows:

Suppose $\phi: X^{n} \rightarrow E^{n}$ is a mapping from an $n$-cell into Euclidean $n$-space. For $y \in E^{n}$ let $S_{\epsilon}(y)$ be the open sphere of radius $\epsilon$ about $y$, and $U_{1}, U_{2}, U_{3}, \cdots$ be those components of $\phi^{-1}\left(S_{6}(y)\right) \equiv W$ having the property that $U_{i} \cap \dot{X}^{n}=0, i=1,2,3, \cdots\left(\dot{X}^{n}\right.$ is the boundary of $\left.X^{n}\right)$.

If $\quad=\phi \mid W$ then $\bar{\phi}: W \rightarrow S_{\mathrm{e}}(y)$ and induces a homomorphism $\bar{\phi}^{*}: H^{n}\left(S_{\epsilon}(y)\right) \rightarrow H^{n}(W)$ between the Lefschetz groups. (See Cartan [1].) But $H^{n}(W)$ is naturally isomorphic to the weak direct product $P w H^{n}\left(U_{i}\right)$. If this isomorphism is denoted by $\eta$, and generators $\mathbf{s}$ and $\boldsymbol{u}_{i}$ are selected for the infinite cyclic groups $H^{n}\left(S_{6}(y)\right)$ and $H^{n}\left(U_{i}\right)$ respectively, $i=1,2,3, \cdots$, then 


$$
\Phi_{\epsilon}^{*}(s)=\left(k_{1} u_{1}, k_{2} u_{2}, k_{3} u_{3}, \cdots\right)
$$

where $k_{i}$ is an integer and different from zero for only a finite number of subscripts $\kappa_{\epsilon}(y)$.

Now $\kappa_{\epsilon}(y)$ is independent of the selection of generators and does not decrease as $\epsilon \rightarrow 0$. If $\kappa(y)=\lim \kappa_{\epsilon}(y)$, then in case $n=2$, Radó defines it to be the essential multiplicity of $y$ under $\phi$. He further defines $V_{\phi}\left(X^{2}\right)=\int_{E^{2}} \kappa(y) d y$ and shows that

$$
L^{2}(\phi)=\int_{E^{2}} \kappa(y) d y .
$$

If $n>2$ then $L^{n}(\phi) \geqq \int_{E^{n}} \kappa(y) d y$ and the equality need not hold even if $n=3$. ( $L^{n}(\phi)$ is the $n$-dimensional Lebesgue "area" of the "surface" represented by $\phi$ and is defined by obvious modifications of \$11.)

On the other hand, Federer defines $\lambda_{\epsilon}(y)=\sum\left|k_{i}\right|$, and $\lambda(y)$ $=\lim \lambda_{\epsilon}(y)$. He then proves the fundamental equality:

$$
L^{n}(\phi)=\int_{E^{n}} \lambda(y) d y \text {. }
$$

The publication of this beautiful result will certainly be awaited with interest.

It should be mentioned, under this first direction of generalization, that results for mappings-even from 3-cells-comparable in scope and importance with those results in the 2-dimensional case which are concerned with middle space topology lead immediately to basic unsolved problems; for example, the characterization of a 3-cell. One is perhaps permitted, therefore, to take a dim view of the possibility of startling developments along the lines of middle space topology in the immediate future.

The second direction along which generalizations may be made, especially insofar as the corresponding representation problem is concerned (see \$16), has been of personal interest during the past few years. This general representation problem has been solved and uses the fundamental work done by Roberts and Steenrod [29] in characterizing the monotone image of a 2-manifold.

This is hardly the place for a discussion of the solution, but it may be of interest to note that a theorem corresponding to the cyclic additivity theorem goes through, and yields a generalization of the theorem of Radó concerning surfaces of zero area. (See \$17.) Specifically, if $f: X \rightarrow E^{3}$ is a mapping from a compact connected 2-manifold and $l m$ is a monotone-light factorization of $f$ with middle space $\mathfrak{X}$ (see 
$\S 15)$, then $L^{2}(f)=0$ if and only if $H^{2}(\mathfrak{X})=0$; or alternately, $L^{2}(f)=0$ if and only if $\operatorname{dim} \mathfrak{X}<2$. A certain "slenderness" of the middle space is thus a necessary and sufficient condition for the Lebesgue area to be zero.

\section{BIBLIOGRAPHY}

1. H. Cartan, Methodes modernes en topologie algebrique, Comment. Math. Helv. vol. 18 (1945) pp. 1-15.

2. Lamberto Cesari, Su di un problema di analysis situs dello spazio ordinario, Rendiconti del Istituto Lombardo di Scienze e Lettere vol. 74 (1941) pp. 267-291.

3. - Caratterizzazione analitica delle superficie continue di area finita secondo Lebesgue, Annali della R. Scuola Normale Superiore di Pisa (2) vol. 10 (1941) pp. 253-294 and vol. 11 (1942) pp. 1-42.

4. - Sui fondamenti geometrici dell' integrale classico per l'area delle superficie in forma parametrica, Memorie Reale Accademia d'Italia vol. 13 (1943) pp. 1323-1481.

5. - Sulle superficie di area finita secondo Lebesgue, Rendiconti Reale Accademia d'Italia (7) vol. 3 (1941) pp. 350-365.

6. - Sul concetto di trasformazione assolutamente continua, Bollettino della Unione Matematica Italiana (2) vol. 5 (1943) pp. 5-10.

7. - Una uguaglianza fondamentale per l'area delle superficie, Atti della Reale Accademia d'Italia vol. 14 (1944) pp. 891-950.

8. - Rappresentazione quasi conforme delle superficie continue, Rendiconti dell'Accademia Nazionale dei Lincei (8) vol. 1 (1946) pp. 509-514.

9. - Sulla rappresentazione delle superficie continue di area finita secondo Lebesgue, Rendiconti del Istituto Lombardo di Scienze e Lettere vol. 79 (1945) pp. $1-31$.

10. S. Eilenberg, On a linkage theorem of L. Cesari, Bull. Amer. Math. Soc. vol. 53 (1947) pp. 1192-1195.

11. M. Fréchet, Sur la distance de deux surfaces, Annales de la Société Polonaise Mathématique vol. 3 (1924) pp. 4-19.

12. Z. de Geöcze, Recherches generales sur la quadrature des surfaces courbes, part I, Math. Naturw. Berichte Ungarn vol. 27 (1909) pp. 1-21; part II, ibid. pp. 131-163; part III, ibid. vol. 30 (1912) pp. 1-29.

13. R. G. Helsel, $A$ theorem on surface area, Trans. Amer. Math. Soc. vol. 61 (1947) pp. 443-453.

14. B. v. Kerékjárt6, Involutions et surfaces continues, Acta Univ. Szeged. vol. 3 (1927) pp. 49-67.

15. H. Lebesgue, Intégrale, longueur, aire, Ann. Mat. Pura Appl. vol. 7 (1902) pp. 231-359.

16. E. J. McShane, On the semi-continuity of double integrals in the calculus of variations, Ann. of Math. vol. 33 (1932) pp. 460-484.

17. C. B. Morrey, Jr., An analytic characterization of surfaces of finite Lebesgue area, part I, Amer. J. Math. vol. 57 (1935) pp. 692-702.

18. T. Rad6, Über das Flachenmass rektifizierbarer Flächen, Math. Ann. vol. 100 (1928) pp. 445-479.

19. - On continuous transformations in the plane, Fund. Math. vol. 27 (1936) pp. 201-211.

20. - On absolutely continuous transformations in the plane, Duke Math. J. vol. 4 (1938) pp. 189-221. 
21. - On the semi-continuity of double integrals in the parametric form, Trans. Amer. Math. Soc. vol. 51 (1942) pp. 336-361.

22. - On continuous path-surfaces of zero area, Ann. of Math. vol. 44 (1943) pp. 173-191.

23. - On continuous mappings of Peano spaces, Trans. Amer. Math. Soc. vol. 58 (1945) pp. 420-454. 106.

24. —- On surface area, Proc. Nat. Acad. Sci. U. S. A. vol. 31 (1945) pp. 102-

25. - Two-dimensional concepts of bounded wariation and absolute continuity, Duke Math. J. vol. 14 (1947) pp. 587-608.

26. - - Length and area, Amer. Math. Soc. Colloquium Publications, vol. 30, 1948.

27. T. Rad6 and P. V. Reichelderfer, $A$ theory of absolutely continuous transformations in the plane, Trans. Amer. Math. Soc. vol. 49 (1941) pp. 258-307.

28. $P$. V. Reichelderfer, On bounded variation and absolute continuity for parametric representations of continuous surfaces, Trans. Amer. Math. Soc. vol. 53 (1943) pp. 251291.

29. J. H. Roberts and N. E. Steenrod, Monotone transformations of two-dimensional manifolds, Ann. of Math. vol. 39 (1938) pp. 851-862.

30. G. T. Whyburn, Analytic topology, Amer. Math. Soc. Colloquium Publications, vol. $28,1942$.

31. J. W. T. Youngs, Curves and surfaces, Amer. Math. Monthly vol. 51 (1944) pp. 1-11.

32. - The topological theory of Fréchet surfaces, Ann. of Math. vol. 45 (1944) pp. 753-785. 673.

33. - On surfaces of class $K_{1}$, Bull. Amer. Math. Soc. vol. 51 (1945) pp. 669-

\section{INDIANA UNIVERSITY}

